\title{
FLUXO DE POTÊNCIA AC PARA OPERAÇÃO INDEPENDENTE DE ÁREAS INTERLIGADAS
}

\author{
Mauricio Granada Echeverri* ${ }^{*}$ \\ granadameieee.org
}

\author{
Marcos J. Rider ${ }^{\dagger}$ \\ mjrider@dee.feis.unesp.br
}

\author{
José R. Sanches Mantovani ${ }^{\dagger}$ \\ mant@dee.feis.unesp.br \\ *Universidad Tecnológica de Pereira \\ Programa de ingeniería eléctrica \\ Pereira, Risaralda, Colombia \\ ${ }^{\dagger}$ Universidade Estadual Paulista - Campus de Ilha Solteira \\ Departamento de Engenharia Elétrica \\ Laboratório de Planejamento de Energia Elétrica, Ilha Solteira, SP, Brasil
}

\begin{abstract}
AC power flow for independent operation of interconnected areas

This paper adjusts a mathematical decomposition approach to the AC power flow problem in power systems with interconnected areas operated by diferent transmission system operators (TSO). The proposed strategy allows finding the operation point of a particular area without explicit knowledge of network data of the other interconnected areas, being only necessary to exchange border information related to the tie-lines between areas. The methodology is based on the decomposition of the first-order optimality conditions of the AC power flow, which is formulated as a nonlinear programming problem. To allow better visualization of the concept of independent operation of each TSO, an artificial neural network have been used to simulate the border information calculation of the interconnected TSOs. A multi-area Power Flow tool can be seen as a basic building block able to address a large number of problems under a multi-TSO competitive market philosophy. The IEEE RTS-96 power system is used in order

Artigo submetido em 27/05/2010 (Id.: 01154) Revisado em 01/09/2010,11/11/2010, 04/01/2011

Aceito sob recomendação do Editor Associado Prof. Antonio Carlos Zambroni de Souza
\end{abstract}

to show the operation and effectiveness of the decentralized AC Power Flow.

KEYWORDS: Multi-area power systems, decomposition methods, decentralized coordination, power flow, neural networks.

\section{RESUMO}

Neste artigo utiliza-se um método de decomposição matemática para resolver um problema de fluxo de carga AC em sistemas de potência compostos de áreas interligadas e administradas por diferentes operadores regionais do sistema elétrico (ORS). A estratégia proposta permite encontrar o ponto de operação de uma determinada área sem conhecer explicitamente os dados da rede das outras áreas interligadas, sendo necessária apenas a troca de informações de fronteira associadas às linhas de interligação. A metodologia baseia-se na decomposição das condições de otimalidade de primeira ordem do fluxo de potência AC que, por sua vez, é formulado como um problema de programação não-linear. Para visualizar melhor o conceito de operação independente de cada ORS, usou-se uma rede neural artificial para simular os cálculos das variáveis de fronteira dos ORS interligados. Um fluxo de carga multi-áreas pode ser visto como uma 
ferramenta básica que pode ser usada no âmbito de mercados competitivos multi-agentes. O sistema de teste IEEE RTS-96 é utilizado para demonstrar o funcionamento e a eficácia do fluxo de potência AC multi-áreas.

PALAVRAS-CHAVE: Sistemas de potência multi-áreas, métodos de decomposição, coordenação descentralizada,fluxo de potência, redes neurais

\section{INTRODUÇÃO}

Os sistemas de potência reais são geralmente compostos por áreas interligadas, onde cada uma é controlada por um operador regional do sistema (ORS). Geralmente, para obter o ponto de operação de uma área, o operador dessa área deve conhecer todos os dados de rede do sistema completo, ou utilizar uma rede equivalente aproximada para algumas regiões do sistema. Atualmente, com a existência de mercados competitivos, nem sempre é possível ter a informação completa do sistema. Nos mercados elétricos liberalizados, a operação do sistema é fortemente afetada pela concorrência dos participantes no mercado. Por esta razão, cada ORS tem políticas de confidencialidade e segurança que restringem o acesso às informações da rede. Sob este cenário, o conceito de operação descentralizada surge como uma solução que proporciona flexibilidade e robustez aos procedimentos matemáticos usados na coordenação dos sistemas de potência multi-áreas. Este conceito permite uma operação independente para cada ORS sujeita a um processo de coordenação global, para uma operação ótima integrada. A ideia básica consiste em dividir o problema geral em vários subproblemas regionais associados a cada área, onde cada ORS resolve o seu próprio problema de otimização. Posteriormente, um processo iterativo de coordenação global troca informações estratégicas entre as áreas.

Neste artigo apresenta-se um modelo regional do problema de fluxo de potência AC (FP-AC) descentralizado para sistemas de potência com áreas interligadas. Este modelo regional permite encontrar o ponto de operação de uma determinada área sem conhecer explicitamente os dados de redes das outras áreas interligadas, sendo necessárias apenas as trocas de informações de fronteiras associadas às linhas de interligação (LI).

O FP-AC é modelado como um problema de programação não-linear, de modo que o modelo resultante possa ser decomposto em torno das LI utilizando um esquema de duplicação de variáveis. Posteriormente, é obtido um modelo regional utilizando a decomposição das condições de otimalidade de primeira ordem (DCOP). Este modelo é resolvido iterativamente por cada ORS.
Para fins comparativos e para permitir uma melhor visualização do conceito de operação independente de cada ORS, uma rede neural artificial é usada como uma ferramenta auxiliar no cálculo das informações de fronteiras dos ORS interligados e validar a metodologia proposta.

O sistema teste IEEE RTS-96 é utilizado para demonstrar a robustez e a eficiência da metologia proposta de fluxo de potência AC multi-áreas.

\section{REVISÃO DA LITERATURA}

Com referência às metodologias que visam resolver um FP-AC de forma descentralizada, é possível classificar as técnicas de solução encontradas na literatura em dois grupos: i) aquelas que utilizam os equivalentes de rede e ii) as que utilizam técnicas de decomposição matemática. Relacionado com as técnicas do primeiro grupo, em Haibo et al. (2006) é proposto um algoritmo distribuído para análise de segurança estática de um sistema multi-área. $\mathrm{O}$ equivalente de Thévenin é iterativamente ajustado para reduzir o erro entre o sistema equivalente e o sistema original. Uma barra de referência é definida para cada área do sistema e, consequentemente, é necessário utilizar um processo de sincronização dos ângulos entre as áreas. Em Phulpin et al. (2009) é apresentada uma avaliação dos diferentes tipos de equivalentes de rede para a implementação de um esquema de controle descentralizado de sistemas multi-áreas. Uma implementação do método de particionamento MATE (Multi-Area Thévenin Equivalents) para a solução em paralelo de sistemas de potência é descrita em Tomim et al. (2009). Neste algoritmo as redes elétricas são particionadas em várias subredes interligadas apenas por um número limitado de linhas de transmissão. Neste contexto, cada sub-rede é calculada independentemente e cada solução combinada entre as diferentes áreas. Uma vez que as correntes nas interligações são calculadas, novas injeções de corrente são obtidas para cada subsistema, o que permite a solução final de cada subsistema, levando em consideração suas conexões externas.

Um dos principais pontos de discussão quando se utiliza uma rede equivalente é que o desempenho do processo de otimização é altamente dependente do tipo de equivalente escolhido. Além disso, vários tipos de equivalentes de rede podem ser encontrados para um sistema e diferentes procedimentos de ajuste são necessários para reduzir os erros introduzidos pelas aproximações de cada equivalente.

As técnicas de decomposição matemáticas em que o problema de FP multi-áreas é resolvido possuem poucas referências na literatura especializada. A maioria delas são aplicadas ao problema de fluxo de potência ótimo (Bakirtzis e Biskas, 2003; Biskas e Bakirtzis, 2006; Conejo e Aguado, 
1998; Granada et al., 2008; Kim e Baldick, 1997; Kim et al., 2001; Nogales et al., 2003) . Em Bakirtzis e Biskas (2002) é apresentado um FP-DC descentralizado usando o princípio do problema auxiliar (PPA), no qual um dos objetivos é preservar a confidencialidade dos dados de rede para cada ORS em um mercado de livre concorrência.

\section{FP-AC COMO UM PROBLEMA DE PROGRAMAÇÃO NÃO LINEAR}

Para utilizar as ferramentas matemáticas para decompor problemas de programação não-linear (PNL) e aplicá-las à decomposição do problema de FP-AC, o primeiro passo é formular o problema adequadamente. O problema de FP-AC pode ser formulado como um problema de PNL, onde a função objetivo consiste em minimizar a soma dos erros de potência ao quadrado das equações de balanço nodal (Iwamoto e Tamura, 1981; Sasson, 1969; Sasson et al., 1971), como mostrado em (1):

$$
\min \quad f(\mathbf{X})=\frac{1}{2}\left([\mathbf{g}(\mathbf{X})]^{T} \cdot \mathbf{g}(\mathbf{X})\right)
$$

onde $\mathbf{g}(\mathbf{X})$ é o conjunto de restrições de igualdade:

$$
\mathbf{g}(\mathbf{X})=\left\{\begin{array}{cl}
P_{k}(\mathbf{X})-P_{N_{k}}=0 & \forall k \in\{\mathbf{P V} \cup \mathbf{P Q}\} \\
Q_{k}(\mathbf{X})-Q_{N_{k}}=0 & \forall k \in\{\mathbf{P Q}\}
\end{array}\right.
$$

PQ é um vetor de índices das barras de carga e $\mathbf{P V}$ é um vetor de índices das barras de tensão controlada. $\mathbf{X}$ é o vetor de variáveis não especificadas $\left(\mathbf{X}=[\mathbf{V}, \theta]^{\mathbf{T}}\right), \mathbf{V}$ e $\theta$ são os vetores das magnitudes e ângulos das tensões, respectivamente (variáveis de estado do problema). $P_{k}$ e $Q_{k}$ são as injeções de potências ativa e reativa calculadas na barra $k$, que são uma função das variáveis não especificadas. $P_{N_{k}}$ e $Q_{N_{k}}$ são a potência ativa e reativa líquidas injetadas na barra $k$, respectivamente.

A abordagem do problema de FP através da PNL pode ser uma ferramenta importante para estudos de estabilidade e colapso de tensão e para obter convergência para problemas de fluxo de potência de sistemas mal-condicionados, entre outros (Iwamoto e Tamura, 1981).

\section{ESQUEMA DE DECOMPOSIÇÃO}

Uma condição necessária para a decomposição trivial de um problema de PNL em subproblemas menores é que tanto a função objetivo como as restrições do problema sejam separáveis. Ou seja, as variáveis de cada subproblema resultante do processo de decomposição, não podem estar associadas aos outros subproblemas. Com o objetivo de buscar uma decomposição trivial do problema de FP-AC e obter um modelo equivalente onde as variáveis de fronteira (VF) sejam claramente definidas, é implementado um esquema de separação em torno das LI que ligam as diferentes áreas do sistema multi-áreas, como ilustrado na Figura 1.

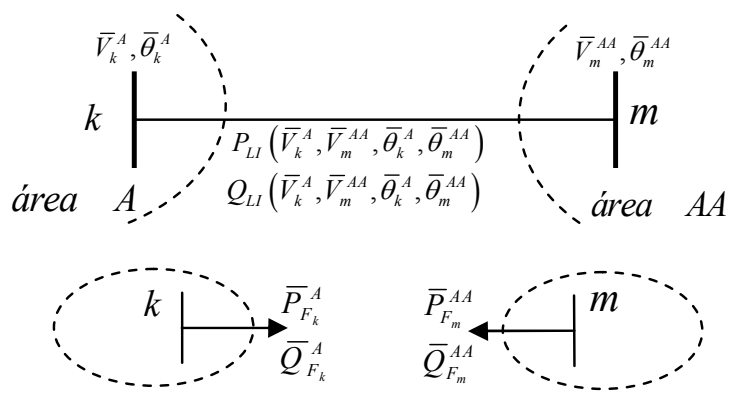

Figura 1: Esquema de separação.

A decomposição é feita usando geradores fictícios que representam os fluxos através das LI. Quatro novas VF por LI são adicionadas ao problema original, $P_{F_{k}}^{A}, Q_{F_{k}}^{A}$, $P_{F_{m}}^{A A}$ e $Q_{F_{m}}^{A A}$, como ilustrado na Figura 1. Essas variáveis representam as gerações fictícias necessárias para a viabilidade dos problemas nas áreas $A$ e $A A$, respectivamente. $\mathrm{O}$ conjunto de restrições $\mathbf{g}\left(\mathbf{X}^{A}\right)$ também se aplica às barras de fronteira, considerando que se houver geração conectada na barra de fronteira, essa barra é classificada como $P V$, caso contrário, é classificada como barra $P Q$. O problema (1) pode ser presentado de forma equivalente, com as variáveis de fronteira explícitas, como segue:

$$
\begin{aligned}
& \min \sum_{A}\left(f\left(\mathbf{X}^{A}\right)\right) \\
& s . a \\
& {\left[P_{F_{k}}^{A}\right]-\left[P_{L I_{k m}}\left(\mathbf{X}^{A}, \mathbf{X}^{A A}\right)\right]=0} \\
& {\left[Q_{F_{k}}^{A}\right]-\left[Q_{L I_{k m}}\left(\mathbf{X}^{A}, \mathbf{X}^{A A}\right)\right]=0} \\
& \quad k \in \mathbf{B B}^{A} ; m \in \mathbf{B B}^{A A} ; \forall A
\end{aligned}
$$

Onde $P_{L I_{k m}}$ e $Q_{L I_{k m}}$ são vetores de potências calculadas (ativa e reativa) fluindo entre as barras $k$ e $m$ das áreas interligadas. $\mathrm{O}$ vector $\mathbf{B B}^{A}$ representa o conjunto de barras de fronteira na área $A$.

As restrições (4) e (5), conhecidas como restrições de acoplamento, são necessárias para coordenar o processo de otimização global e garantir que no ponto ótimo o problema (1) seja equivalente ao problema (3)-(5). Essas restrições devem ser aplicadas em todas as barras de fronteira. Devido ao fato de que as restrições de acoplamento são funções de variáveis que pertencem às áreas adjacentes, o problema 
(3)-(5) não pode ser separado de forma direta ou trivial, sendo necessário o uso de um método matemático de decomposição, como mostrado a seguir.

\subsection{Problema regional}

O algoritmo de decomposição utilizado neste trabalho, para obter um modelo regional do FP-AC, baseia-se na decomposição das condições de otimalidade para o problema centralizado (3)-(5). Este algoritmo pode ser interpretado como uma aplicação particular do processo de relaxação lagrangiana (Nogales et al.; 2003). A ideia básica é obter o mesmo ponto de operação que seria alcançado com um fluxo de potência centralizado tradicional. Esta decomposição é realizado de tal maneira que a união de todas as condições de otimalidade de primeira ordem de Karush-Kuhn-Tucker de todos os problemas regionais na k-ésima iteração de um processo de coordenação global é idêntica ao conjunto de condições de otimalidade de primeira ordem do problema global original (Conejo et al.; 2006, 2002). Neste processo iterativo, os valores das variáveis correspondentes às barras de fronteira de uma área adjacente são conhecidos da iteração anterior (variáveis indicadas pela sobrelinha). Assim, utilizando o esquema de decomposição proposto por Conejo et al. (2002), o problema de PNL para a área $A$ é:

$$
\begin{aligned}
& \min _{\mathbf{X}^{A}, \mathbf{S}_{F}^{A}} \quad f\left(\mathbf{X}^{A}\right)+\left[\overline{\mathbf{u}}^{A A}\right]^{T} \mathbf{S}_{F}^{A} \\
& \text { s.a } \\
& \mathbf{g}_{F}\left(\mathbf{X}^{A}, \mathbf{S}_{F}^{A}\right)=\mathbf{S}_{F}^{A}-\mathbf{S}_{L I}\left(\mathbf{X}^{A}, \overline{\mathbf{X}}^{A A}\right)=0: \mathbf{u}^{A}
\end{aligned}
$$

Onde $f$ é a função objetivo do problema regional. O vetor $\mathbf{g}_{F}$ representa o conjunto de restrições (4) e (5), associadas às gerações fictícias nas barras de fronteira. $\mathrm{O}$ segundo termo da função objetivo pode ser reescrito da seguinte forma:

$$
\left[\overline{\mathbf{u}}^{A A}\right]^{T}\left[\mathbf{S}_{F}^{A}\right]=\left[\overline{\mathbf{a}}_{L I}^{A A}\right]^{T}\left[\mathbf{P}_{F}^{A}\right]+\left[\overline{\mathbf{r}}_{L I}^{A A}\right]^{T}\left[\mathbf{Q}_{F}^{A}\right]
$$

Os multiplicadores de Lagrange $\mathbf{u}^{A}$, das restrições de acoplamento, são sensibilidades que relacionam a variação da função objectivo com a variação da potência ativa e reativa exportada da área $A$ para outras áreas adjacentes $A A$. Estes multiplicadores são utilizados por outras áreas como as sensibilidades associadas à importação de potência ativa $\mathrm{e}$ reativa, caso em que eles são conhecidos da iteração anterior e são indicados como $\overline{\mathbf{a}}_{L I}^{A A}$ e $\overline{\mathbf{r}}_{L I}^{A A}$, respectivamente.

\section{PROCESSO DE COORDENAÇÃO GLOBAL}

Devido ao fato que os resultados obtidos a partir do FP-AC descentralizado são de especial interesse para uma determinada área $A$, o ORS desta área específica $\left(O R S_{A}\right)$ será o responsável pela realização do processo de coordenação. Esse processo consiste de três etapas iterativas: i) distribuir para cada área do sistema global os valores atuais das variáveis de sua fronteira, ii) receber as respostas (estado) de cada área em função das variáveis de fronteira previamente distribuídas (magnitude e ângulo da tensão nas barras de fronteira, fluxos nas LI e multiplicadores de Lagrange) e iii) verificar os critérios de convergência. Assim, o processo de coordenação global pode ser visto, essencialmente, como um processo de distribuição e recepção de informações de fronteiras, realizado por parte do operador da área sob análise.

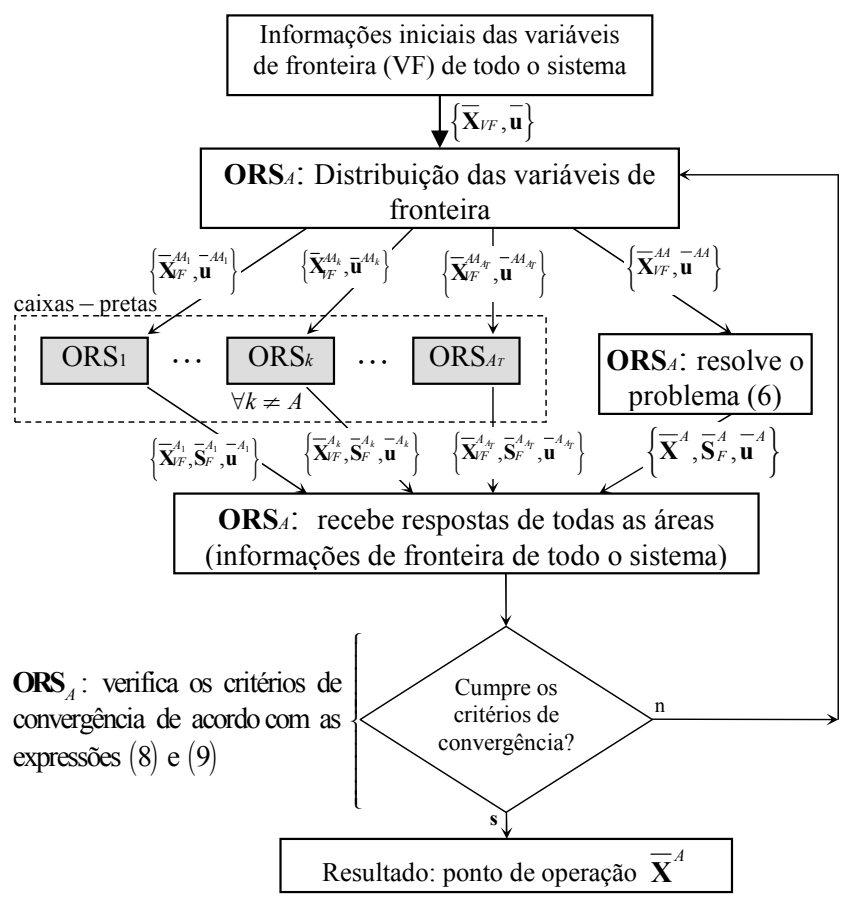

Figura 2: Processo de coordenação conduzido pelo $O R S_{A}$.

A Figura 2 ilustra o processo de coordenação global conduzido pelo $O R S_{A}$ para um sistema com $A_{T}$ áreas. $A A_{k}$ representa o conjunto das áreas adjacentes à área $A_{k}$ e $\mathbf{X}_{V F}^{A_{k}}$ representa o vetor de tensões (magnitude e ângulo) nas barras de fronteira da área $A_{k}$. Os três blocos básicos de construção são explicadas a seguir.

Distribuição das variáveis de fronteira: Inicialmente, na primeira etapa, os valores das VF podem ser escolhidos convenientemente para executar a primeira iteração do processo, por exemplo; as tensões iguais a 1 pu e os ângulos, multiplicadores de Lagrange e fluxos nas LI iguais a zero. As informações de fronteira de todo o sistema é processada pelo $O R S_{A}$ para iniciar o processo de troca de variáveis de fronteira, de modo que cada área receba as informações 
apenas de suas fronteiras. $\mathrm{O} O R S_{A}$ deve resolver seu próprio problema regional, considerando apenas as variáveis de suas próprias fronteiras, de acordo com (6).

Receber as respostas de todas as áreas: Os outros ORS interligados, diferente do $O R S_{A}$, são representados como "caixas-pretas", onde não é necessário conhecer suas configurações internas, apenas os valores das variáveis nas barras de fronteira. Contudo, para alcançar a convergência global usando o esquema descentralizado, é necessário que as respostas dessas caixas-pretas sejam determinadas usando o modelo (6). As respostas (variáveis de fronteira) devem ser calculadas e disponibilizadas em tempo real pelos outros ORS, por isso não é necessário que o $O R S_{A}$ conheça informações adicionais de outras áreas. Observe também que o único ORS que tem informações explícitas sobre as variáveis de estado é o $O R S_{A}$ (vector de variáveis de estado $\overline{\mathbf{X}}^{A}$ ), de modo que no final do processo obtém-se o ponto de operação somente na área $A$ (ver Figura 2).

Verificar os critérios de convergência: $\mathrm{O}$ algoritmo de coordenação global finaliza quando as equações (8) e (9) são satisfeitas para cada LI do sistema global. Para todos os pontos de KKT (Karush-Kuhn-Tucker), as equações devem ser satisfeitas com uma tolerância $\varepsilon_{P}$ (MW) e $\varepsilon_{Q}$ (MVAR), respectivamente:

$$
\begin{aligned}
& \Delta P_{k m}=\left|\bar{P}_{F_{k}}^{A}+\bar{P}_{F_{m}}^{A A}-P_{\operatorname{Perd}_{k m}}\left(\bar{V}_{k}^{A}, \bar{V}_{m}^{A A}, \bar{\theta}_{k}^{A}, \bar{\theta}_{k}^{A A}\right)\right| \leq \varepsilon_{P} \\
& \Delta Q_{k m}=\left|\bar{Q}_{F_{k}}^{A}+\bar{Q}_{F_{m}}^{A A}-Q_{\operatorname{Perd}_{k m}}\left(\bar{V}_{k}^{A}, \bar{V}_{m}^{A A}, \bar{\theta}_{k}^{A}, \bar{\theta}_{k}^{A A}\right)\right| \leq \varepsilon_{Q}
\end{aligned}
$$

Essas equações são o resultado da análise dos fluxos de potências ativa e reativa nas LI, considerando as perdas de potências ativa e reativa $\left(P_{\operatorname{Perd}_{k m}}, Q_{\operatorname{Perd}_{k m}}\right)$.

\section{FP DESCENTRALIZADO USANDO REDES NEURAIS}

As redes neurais artificiais (RNA) constituem uma ferramenta adequada para a modelagem de sistemas estáticos não-lineares, uma vez que têm a capacidade inerente de superar a complexidade de modelagem e permitir as implementações em tempo real de processos complexos de cálculo. Em aplicações onde é necessário executar muitos FP-AC, pode ser útil explorar as características das RNA.

Neste artigo, o uso das RNA permite uma melhor visualização do conceito de operação independente de cada ORS, os quais operam como "caixas-pretas", onde não é necessário conhecer suas configurações internas. Assim, uma rede neural artificial é usada como uma ferramenta de cálculo para validar a metodologia proposta. A função da RNA neste trabalho é simular o cálculo das informações de fronteira dos ORS.

\subsection{Arquitetura da RNA}

A RNA deve calcular as variáveis de fronteira de cada $O R S_{k}$, para $\forall k \neq A$. Uma rede neural com a arquitetura mostrada na Figura 3 é usada em cada área $k$ para estimar os valores das VF do $O R S_{k}$, tendo como entrada os valores conhecidos das VF dos ORS vizinhos, incluindo o $O R S_{A}$.

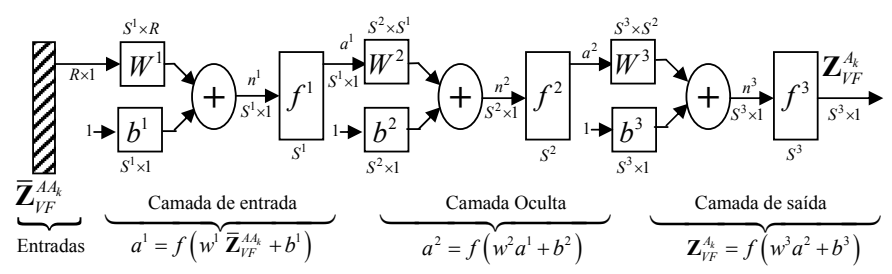

Figura 3: Arquitetura da RNA usada pelo $O R S_{k}$.

Na Figura 3, o vetor de entrada $\overline{\mathbf{Z}}_{V F}^{A A_{k}}$ compreende as variáveis de fronteira das áreas adjacentes da área $k$, ou seja, $\overline{\mathbf{z}}_{V F}^{A A_{k}}=\left[\overline{\mathbf{X}}_{V F}^{A A_{k}}, \overline{\mathbf{u}}^{A A_{k}}\right]^{T}$. Esse vetor é de dimensão $R$, e é definido pela expressão $R=4 n t l_{A_{k}}$; onde $n t l_{A_{k}}$ é o número de LI associadas com a área $A_{k}$, e o número 4 é por considerar quatro tipos de variáveis de entrada por cada LI $\left(\mathbf{V}^{A A}, \theta^{A A}, \mathbf{a}^{A A}, \mathbf{r}^{A A}\right)$. $S^{3}$ é a dimensão do vetor de saída $\mathbf{Z}_{V F}^{A_{k}}$, que por sua vez define o número de neurônios na camada de saída $S^{3}=6 n b b_{A_{k}}$; onde $n b b_{A_{k}}$ é o número de $\mathrm{VF}$ associadas com a área $A_{k}$, e o número 6 é por considerar seis tipos de variáveis de saída para cada barra de fronteira $\left(\mathbf{V}^{A}, \theta^{A}, \mathbf{P}_{F}^{A}, \mathbf{Q}_{F}^{A}, \mathbf{a}^{A}, \mathbf{r}^{A}\right)$.

As funções de ativação $f^{1}$ e $f^{3}$ são funções lineares, e a função da camada oculta $f^{2}$ é considerada uma função não-linear, a tangente hiperbólica. Para selecionar a arquitetura da RNA, foi adotada a regra apresentada por Prechelt (1994). Esta regra estabelece que as arquiteturas sejam definidas mediante a variação do número de neurônios na camada oculta, sendo selecionada a arquitetura que apresentar o melhor desempenho durante os treinamentos sobre o conjunto de validação.

A arquitetura de RNA da Figura 3 correlaciona o vetor de entrada com o vetor de saída através da seguinte equação:

$$
\mathbf{Z}_{V F}^{A_{k}}=f^{3}\left(W^{3} f^{2}\left(W^{2} f^{1}\left(W^{1} \overline{\mathbf{Z}}_{V F}^{A A_{k}}+b^{1}\right)+b^{2}\right)+b^{3}\right)
$$

Para encontrar os pesos $W^{i}$ e $b^{i}(i=1,2,3)$, necessários em (10), deve ser resolvido o problema de treinamento de uma rede de múltiplas camadas. Para isso, é usado o algoritmo 
de backpropagation de Levenberg-Marquardt, apresentado em Hagan e Menhaj (1994), considerando como índice de desempenho o erro quadrático médio.

\subsection{Treinamento da RNA}

O conjunto de dados de treinamento, de uma determinada área $A_{k}$, é composto por um número $n p_{k}$ de padrões de entrada e seus respectivos comportamentos de saída (usam-se pares de entrada-saída conhecidos). O conjunto de padrões é composto por valores aleatórios distribuídos uniformemente dentro de uma faixa cuidadosamente escolhida. A faixa em que cada variável de entrada pode se mover é determinada pela solução do FP-AC descentralizado (6). Este procedimento é executado para todas as áreas em cada iteração do processo de coordenação. Assim, estabelecem-se os valores mínimos e máximos que podem ser alcançados pelas VF, em cada área.

O conjunto de dados de saída é construído através da resolução do problema (6) para cada um dos padrões aleatórios, estabelecidos para cada área. Para um melhor desempenho da rede, o conjunto de treinamento deve também conter as condições iniciais das variáveis de fronteiras, porque esta é uma informação importante no desempenho do FP-AC multi-áreas.

\section{RESULTADOS}

Nesta seção apresentam-se os resultados obtidos de testes com a metodologia proposta através do o sistema IEEE RTS-96 com três áreas (ver Apêndice A), adotando-se o $O R S_{3}$ como coordenador do processo descentralizado.

Os resultados mostram que o ponto de operação do sistema, obtido pelo FP-AC centralizado, é o mesmo que o obtido pelo FP-AC descentralizado para a área 3. Ou seja, manteve-se a operação independente de cada área, porque as informações internas de cada área não precisaram ser partilhadas (a informação é mantida confidencial).

Os resultados do sistema descentralizado correspondem aos dois tipos de abordagem, utilizando o modelo regional (MR) e utilizando RNA. Foi desenvolvido um programa no software MATLAB 7.0 usando a função fmincon, e foi utilizado um computador Pentium de 1.8G (2G RAM).

Na figura 4 mostram-se os valores finais das variáveis de fronteira e os fluxos de potência nas linhas 123-217 e 223-318, obtidos resolvendo o MR. Estas informações de fronteira, em todas as linhas de ligação do sistema, permitem ao $O R S_{3}$ verificar o cumprimento dos critérios de convergência do FP-AC descentralizado.

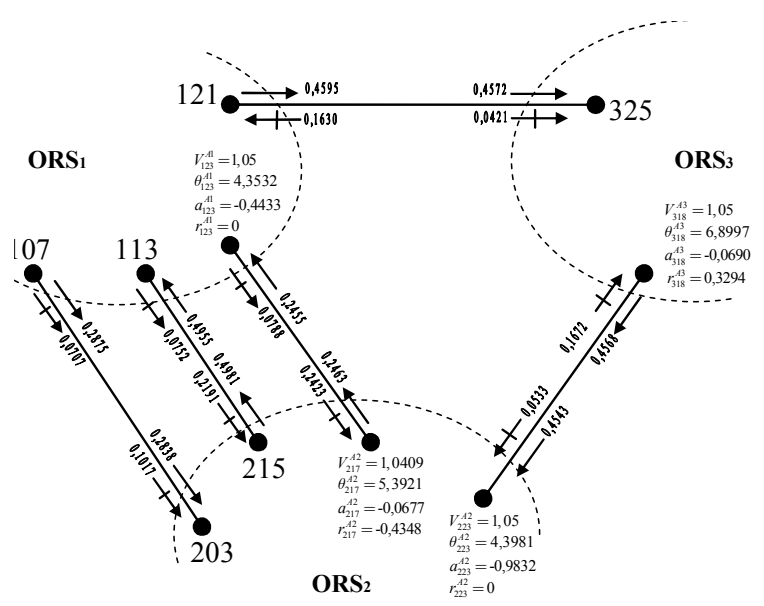

Figura 4: valores finais das variáveis de fronteira.

Os valores dos fluxos de potência nas LI encontrados utilizando o MR, as RNA e o FP-AC centralizado, são bastante semelhantes.

Resolvendo o MR é possível alcançar um maior grau de precisão considerando os erros $\varepsilon_{P}$ e $\varepsilon_{Q}$ pequenos o suficiente, o que leva a um maior número de iterações do processo de coordenação global e, consequentemente, maior tempo de processamento. Para melhorar o grau de precisão usando as RNA, pode ser considerado um maior número de padrões $n p$ e/ou um número maior de neurônios na camada oculta e/ou um maior número de épocas, o que leva ao aumento do tempo computacional no processo de treinamento da RNA. Neste caso, considerou-se que $\varepsilon_{P}=$ $\varepsilon_{Q}=0,01$ p.u.

A arquitetura da rede neural usada para a área 1 é igual à utilizada na área 2 e foi selecionada por meio de testes na camada oculta (Prechelt; 1994) . Assim, foi utilizado um número de padrões $n p=100,100$ épocas, 24 neurônios na camada de entrada $\left(S^{1}=24\right)$ e 72 neurônios na camada oculta $\left(S^{2}=72\right)$.

Os comportamentos dos erros $\Delta P_{k m}$ e $\Delta Q_{k m}$, em duas LI (123-217 e 223-318), são mostrados nas Figuras 5 e 6, respectivamente. Todas as LI do sistema apresentam um comportamento de convergência decrescente e atingem erros de potência ativa e reativa com níveis aceitáveis de precisão.

As linhas pontilhadas mostram a evolução dos erros de potência ao resolver o MR. As linhas contínuas mostram o mesmo processo usando RNA. As Figuras 5 e 6 mostram que a RNA tem uma capacidade adequada de generalização.

A Tabela 1 apresenta o ponto de operação (magnitude e ângulo da tensão ) da área 3, utilizando o esquema centralizado (colunas 2 e 3 , respectivamente). Os pontos 

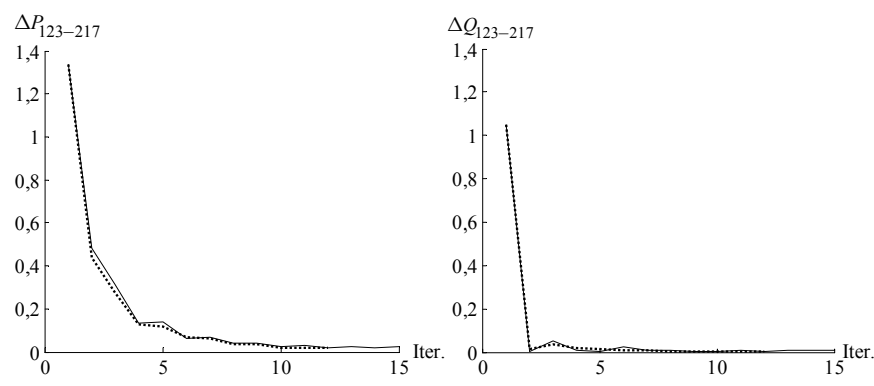

Tabela 1: Comparação dos pontos de operação obtidos para a área $3\left(\varepsilon_{P}=\varepsilon_{Q}=0,01\right)$.

Figura 5: Evolução de $\Delta P$ e $\Delta Q$ na LI 123-217.

\begin{tabular}{|c|c|c|c|c|c|c|}
\hline \multirow[b]{2}{*}{ Barra $i$} & \multicolumn{2}{|c|}{$\begin{array}{c}\text { FP-AC } \\
\text { Centralizado }\end{array}$} & \multicolumn{2}{|c|}{$\begin{array}{c}\text { FP-AC } \\
\text { descentralizado } \\
\text { (modelo regional) }\end{array}$} & \multicolumn{2}{|c|}{$\begin{array}{c}\text { FP-AC } \\
\text { descentralizado } \\
\text { (RNA) }\end{array}$} \\
\hline & $\overline{V_{i}}$ & $\overline{\overline{\theta_{i}}}$ & $\overline{\theta_{i}}$ & $\overline{\nu \Delta \theta_{i}}$ & $\overline{\theta_{i}}$ & $\overline{\nu \Delta \theta_{i}}$ \\
\hline 301 & 1,0350 & $-11,8660$ & $-11,8720$ & 0,0060 & $-11,8680$ & 0,0020 \\
\hline 302 & 1,0350 & $-11,9160$ & $-11,9230$ & 0,0070 & $-11,9180$ & 0,0020 \\
\hline 303 & 0,9889 & $-10,9000$ & $-10,9070$ & 0,0070 & $-10,9030$ & 0,0030 \\
\hline 304 & 1,0009 & $-13,4070$ & $-13,4130$ & 0,0060 & $-13,4090$ & 0,0020 \\
\hline 305 & 1,0313 & $-13,6550$ & $-13,6610$ & 0,0060 & $-13,6570$ & 0,0020 \\
\hline 306 & 1,0840 & $-16,0440$ & $-16,0500$ & 0,0060 & $-16,0450$ & 0,0010 \\
\hline 307 & 1,0250 & $-5,0405$ & $-5,0465$ & 0,0060 & $-5,0420$ & 0,0015 \\
\hline 308 & 0,9979 & $-10,9910$ & $-10,9970$ & 0,0060 & $-10,9920$ & 0,0010 \\
\hline 309 & 1,0067 & $-10,4780$ & $-10,4840$ & 0,0060 & $-10,4800$ & 0,0020 \\
\hline 310 & 1,0537 & $-12,3230$ & $-12,3290$ & 0,0060 & $-12,3250$ & 0,0020 \\
\hline 311 & 0,9960 & $-5,2247$ & $-5,2308$ & 0,0061 & $-5,2264$ & 0,0017 \\
\hline 312 & 1,0106 & $-4,0550$ & $-4,0605$ & 0,0055 & $-4,0559$ & 0,0009 \\
\hline 313 & 1,0200 & $-0,2671$ & $-0,2726$ & 0,0055 & $-0,2680$ & 0,0009 \\
\hline 314 & 0,9800 & $-3,3246$ & $-3,3315$ & 0,0069 & $-3,3272$ & 0,0026 \\
\hline 315 & 1,0140 & 2,8692 & 2,8611 & 0,0081 & 2,8649 & 0,0043 \\
\hline 316 & 1,0170 & 2,4922 & 2,4846 & 0,0076 & 2,4887 & 0,0035 \\
\hline 317 & 1,0393 & 5,9419 & 5,9329 & 0,0090 & 5,9366 & 0,0053 \\
\hline 318 & 1,0500 & 6,8997 & 6,8908 & 0,0089 & 6,8943 & 0,0054 \\
\hline 319 & 1,0231 & 1,9038 & 1,8976 & 0,0062 & 1,9020 & 0,0018 \\
\hline 320 & 1,0382 & 3,3078 & 3,3029 & 0,0049 & 3,3076 & 0,0002 \\
\hline 321 & 1,0500 & 7,8130 & 7,8038 & 0,0092 & 7,8075 & 0,0055 \\
\hline 322 & 1,0500 & 12,6490 & 12,6400 & 0,0090 & 12,6430 & 0,0060 \\
\hline 323 & 1,0500 & 4,7714 & 4,7672 & 0,0042 & 4,7721 & $-0,0007$ \\
\hline 324 & 0,9881 & $-2,2221$ & $-2,2297$ & 0,0076 & $-2,2257$ & 0,0036 \\
\hline 325 & 1,0504 & 4,9852 & 4,9817 & 0,0035 & 4,9868 & $-0,0016$ \\
\hline
\end{tabular}

de operação obtidos resolvendo o MR e utilizando RNA, alcançam os mesmos valores de magnitudes de tensão em comparação com o FP-AC centralizado.

Os ângulos das tensões nodais, obtidos resolvendo o MR, e os erros com relação ao esquema centralizado, são apresentados nas colunas 4 e 5 , respectivamente. A mesma informação é apresentada nas colunas 6 e 7, para o caso quando se utiliza a RNA. Embora neste caso, o esquema usando RNA apresente resultados melhores do que os obtidos com o MR, esta situação só ocorre com níveis relativamente baixos de precisão $\left(\varepsilon_{P}=\varepsilon_{Q}=0,01\right)$. Para maiores graus de precisão, o esquema usando RNA apresenta um maior desvio do erro na magnitude e no ângulo das tensões, em comparação com o sistema centralizado. Quando o grau de precisão é de $\varepsilon_{P}=\varepsilon_{Q} \leq 0,00001$ o esquema usando RNA não converge, sendo esta uma das principais deficiências na utilização de RNA. Assim, a RNA é usada apenas para proporcionar uma melhor visualização do conceito de operação independente de cada ORS.

Estratégias mistas podem ser implementadas, onde a RNA é utilizada, inicialmente, com um menor grau de precisão e, em seguida, o MR é utilizado para completar o processo de coordenação global. Assim, o tempo computacional total é reduzido e o grau de precisão pode ser melhorado.

$\mathrm{Na}$ Tabela 2 apresentam-se os tempos computacionais obtidos, para diferentes graus de precisão, para convergência

Outros testes foram realizados com a implementação

do algoritmo. O FP-AC centralizado tradicional requer $0,35313 s$ para convergir, com uma precisão de 0,0001 p.u. (Coluna 2). O FP-AC descentralizado utilizando o MR atinge o mesmo grau de precisão que o esquema centralizado em 28 iterações do processo de coordenação global e requer $2,19219 s$ para convergência. Para alcançar uma precisão de 0, $0001 \mathrm{pu}$, usando RNA, foi necessário aumentar o número de neurônios na camada oculta $\left(S^{2}=98\right)$ e o número de padrões de treinamento $n p=200$, conforme mostrado nas colunas 9 e 10, respectivamente. Os tempos de treinamento da RNA para cada caso e para cada área são apresentados nas colunas 7 e 8 . O esquema descentralizado usando RNA exige 39 iterações (coluna 6) e 1,292 segundos para atingir os critérios de convergência com $\varepsilon_{P}=\varepsilon_{Q}=0,01$.

Tabela 2: Tempos computacionais.

\begin{tabular}{|c|c|c|c|c|c|c|c|c|c|}
\hline \multirow[b]{3}{*}{$\varepsilon_{P}=\varepsilon_{Q}$} & \multirow{3}{*}{$\begin{array}{c}\text { FP-AC } \\
\text { centralizado } \\
\text { tempo (s) }\end{array}$} & \multirow{2}{*}{\multicolumn{2}{|c|}{$\begin{array}{c}\text { FP-AC } \\
\text { descentralizado } \\
\text { (modelo regional) }\end{array}$}} & \multicolumn{6}{|c|}{ FP-AC descentralizado (RNA) } \\
\hline & & & & \multirow[b]{2}{*}{ tempo $(\mathrm{s})$} & \multirow[b]{2}{*}{ iter } & \multicolumn{2}{|c|}{$\begin{array}{l}\text { Tempo (s) } \\
\text { treinamento }\end{array}$} & \multirow[b]{2}{*}{$n p$} & \multirow[b]{2}{*}{$S^{2}$} \\
\hline & & tempo $(\mathrm{s})$ & iter & & & $\mathrm{TSO}_{1}$ & $\mathrm{TSO}_{2}$ & & \\
\hline 0,01 & \multirow{3}{*}{0,35313} & 0,9844 & 12 & 0,4813 & 15 & 152 & 287 & 100 & 72 \\
\hline 0,001 & & 1,5063 & 19 & 0,5212 & 23 & \multirow{2}{*}{643} & \multirow{2}{*}{937} & \multirow{2}{*}{200} & \multirow{2}{*}{98} \\
\hline 0,0001 & & 2,19219 & 28 & 1,292 & 39 & & & & \\
\hline
\end{tabular}


computacional da metodologia proposta através do sistema IEEE RTS-96 para avaliar a sua eficiência. O $O R S_{1}$ e o $O R S_{2}$ também foram considerados como coordenadores do sistema descentralizado. Foram também realizados testes com subtrações das atuais LI e com adições de novas LI para testar diferentes configurações do sistema. Para cada nova configuração do sistema uma nova estrutura e um novo treinamento da RNA devem ser considerados. Em todos os casos, a abordagem proposta alcançou a convergência com $\varepsilon_{P}=\varepsilon_{Q}=0,01$.

\section{CONCLUSÕES E RECOMENDAÇÕES}

Neste trabalho apresenta-se um FP-AC descentralizado para sistemas multi-áreas que preserva a confidencialidade dos dados da rede de cada área e permite o funcionamento independente de cada ORS. A abordagem utilizada é fácil de implementar, não requer ajustes de parâmetros, fornece resultados practicamente iguis aos do esquema centralizado e requer a especificação apenas de uma barra de referência em uma das áreas do sistema. Apenas informações das barras de fronteira são trocadas entre cada área.

O problema de FP-AC descentralizado, abordado conforme a proposta deste trabalho através de técnicas de PNL, é uma ferramente básica para ser utilizada no planejamento da operação de sistemas eletricos de potência em cenários de carga pesada e/ou com problemas de reativos e estabilidade de tensão, onde o FP convencional não alcança solução.

Todos os testes efetuados, utilizando critérios de convergência $\varepsilon_{P}=\varepsilon_{Q}=0,01$, o FP-AC descentralizado, utilizando o MR ou RNA, convergiu com sucesso e os resultados alcançaram o mesmo nível de precisão que os obtidos usando um FP-AC tradicional. Para maiores graus de precisão, o esquema usando RNA apresenta um maior desvio do erro na magnitude e ângulo das tensões, em comparação com o esquema usando o MR. Quando o grau de precisão é de $\varepsilon_{P}=\varepsilon_{Q} \leq 0,00001$, o esquema usando RNA não converge. No entanto, as RNA permitem uma melhor visualização do conceito de operação independente de cada ORS, os quais operam como "caixas-pretas", onde não é necessário conhecer suas configurações internas e que só podem ser acessadas em suas fronteiras para obter as variáveis de controle.

Estratégias mistas podem ser implementadas para tornar possível a aplicação da metodologia em tempo real e tirar proveito da velocidade superior das RNA, em relação aos métodos convencionais de solução de problemas não-lineares. Por exemplo, podería ser implementada uma estratégias onde, inicialmente, a RNA é utilizada com um menor grau de precisão e, em seguida, utilizar o esquema de MR para completar o processo de coordenação global.
Assim, o tempo computacional total é reduzido e o grau de precisão pode ser melhorado.

Quando existem mudanças na configuração da rede, como por exemplo uma contingência, a metodologia proposta, usando explicitamente o problema regional (6) em cada uma das áreas conforme apresentado na seção 5, pode ser aplicada para obter o novo ponto de operação do sistema. As informações de fronteira, incluindo os multiplicadores de Lagrange associados as equações de balanço nas LI, fornecem a possibilidade de analisar, por exemplo, as mudanças de fluxos de energia entrando e saindo da área sob análise devido a uma contingência em outra área. Estas informações são importantes em estudos tais como tarifação do tipo pedágio (wheeling) em sistemas de potência (Zhu; 2009).

Quanto à substituição do modelo regional (6) por uma RNA, conforme apresentado na seção 6, esta metodologia é eficiente apenas em redes cuja configuração não muda. Isso ocorre porque a atual arquitetura, mostrada na figura 3 , não tem a capacidade de correlacionar as possíveis mudanças da rede com as variáveis de fronteira. Portanto, para cada mudança da rede numa área, a RNA associada requer um novo treinamento, o que torna inviável as RNA neste contexto. No entanto, uma nova estrutura de RNA que correlaciona as diferentes contingências com as variáveis de fronteira pode ser concebida e estudada em trabalhos futuros.

\section{A SISTEMA DE TESTE IEEE RTS-96}

Os dados de linha e das cargas são extraídos do sistema original apresentado em Grigg et al. (1999). Os dados utilizados das barras de geração são apresentados na Tabela 3.

\section{AGRADECIMENTOS}

Os autores agradecem o apoio da CAPES-Brasil (processo BEX 1202/06-5), da Universidad Tecnológica de Pereira (Colômbia) e do CNPQ-Brasil (processo 302272/2009-7).

\section{REFERÊNCIAS}

Bakirtzis, A. and Biskas, P. (2002). Decentralised DC load flow and applications to transmission management, IEE Proc.-Gener. Trans. Distrib. 149(5): 600-606.

Bakirtzis, A. and Biskas, P. (2003). A decentralized solution to the DC-OPF of interconnected power systems, IEEE Trans. Power App. Syst. 18(3): 1007-1013.

Biskas, P. and Bakirtzis, A. (2006). Decentralized OPF of 
Tabela 3: Dados das barras de geração do sistema teste.

\begin{tabular}{llllll} 
Barra $i$ & $\mathrm{~V}_{i}$ & $\begin{array}{c}\mathrm{P}_{\mathrm{G}} \\
\mathrm{MW}\end{array}$ & $\begin{array}{c}\mathrm{Q}_{\mathrm{G}} \\
\mathrm{MVA}\end{array}$ & $\begin{array}{c}\mathrm{P}_{\mathrm{DW}} \\
\mathrm{MW}\end{array}$ & $\begin{array}{c}\mathrm{Q}_{\mathrm{D}} \\
\text { MVAR }\end{array}$ \\
\hline $\mathbf{2}$ & 1,035 & 150,6 & 34,8 & 108 & 22 \\
7 & 1,035 & 150,6 & 20 & 97 & 20 \\
$\mathbf{1 3}$ (Ref.) & 1,025 & 300 & 19 & 125 & 25 \\
14 & 0,98 & 487 & 148,3 & 265 & 54 \\
15 & 1,014 & 144,1 & 15,1 & 194 & 39 \\
16 & 1,017 & 123,4 & 6,6 & 100 & 64 \\
18 & 1,05 & 380 & 39,7 & 333 & 68 \\
21 & 1,05 & 389,4 & 61,3 & 0 & 0 \\
22 & 1,05 & 256,9 & 18,5 & 0 & 0 \\
23 & 1,05 & 507,9 & 106,3 & 0 & 0 \\
25 & 1,035 & 150,6 & 34,8 & 108 & 22 \\
26 & 1,035 & 150,6 & 20 & 97 & 20 \\
31 & 1,025 & 300 & 19 & 125 & 25 \\
37 & 1,02 & 487 & 148,3 & 265 & 54 \\
38 & 0,98 & 0 & 15 & 194 & 39 \\
39 & 1,014 & 144,1 & 13,1 & 17 & 64 \\
40 & 1,017 & 123,4 & 6,6 & 100 & 20 \\
42 & 1,05 & 380 & 39,7 & 333 & 68 \\
45 & 1,05 & 389,4 & 61,3 & 0 & 0 \\
46 & 1,05 & 256,9 & 18,5 & 0 & 0 \\
47 & 1,05 & 507,9 & 106,3 & 0 & 0 \\
49 & 1,035 & 150,6 & 34,8 & 108 & 22 \\
50 & 1,035 & 150,6 & 20 & 97 & 20 \\
55 & 1,025 & 300 & 19 & 125 & 25 \\
61 & 1,02 & 487 & 148,3 & 265 & 54 \\
62 & 0,98 & 0 & 15 & 194 & 39 \\
63 & 1,014 & 144,1 & 13,1 & 17 & 64 \\
64 & 1,017 & 123,4 & 6,6 & 100 & 20 \\
66 & 1,05 & 380 & 39,7 & 333 & 68 \\
69 & 1,05 & 389,4 & 61,3 & 0 & 0 \\
70 & 1,05 & 256,9 & 18,5 & 0 & 0 \\
71 & 1,05 & 507,9 & 106,3 & 0 & 0 \\
& & & & &
\end{tabular}

large multiarea power system, IEE Proc.-Gener. Transm. Distrib. 153(1): 99-105.

Conejo, A. and Aguado, J. (1998). Multi-area coordinated decentralized DC optimal power flow, IEEE Trans. Power Syst. 13.

Conejo, A., Castillo, E., Minguez, R. and Garcia-Bertrand, R. (2006). Decomposition techniques in mathematical programming., Engineering and Science Applications. XVI, 541 p. 104 illus., Hardcover, ISBN: 978-3-540-27685-2.

Conejo, P., Nogales, F. and Prieto, F. (2002). A decomposition procedure based on approximate newton directions, in Mathematical programming. Springer-Verlag. .

Granada, M., Rider, M., Mantovani, J. and Shahidehpour, M. (2008). Multi-areas optimal reactive power flow, Proc. Transmission and Distribution Conference and Exposition: Latin America, IEEE/PES .

Grigg, C., Wong, P., Albrecht, P., Allan, R., Bhavaraju, M., Billinton, R., Chen, Q., Fong, C., Haddad, S., Kuruganty, S., Li, W., Mukerji, R., Patton, D., Rau, N., Reppen, D., Schneider, A., Shahidehpour, M. and Singh, C. (1999).
The IEEE reliability test system-1996, IEEE Trans. Power Syst. 14: 1010-1020.

Hagan, M. and Menhaj, M. (1994). Training feedforward networks with the marquardt algorithm, IEEE Tran. Neural Networks 5(6): 989-993.

Haibo, Z., Boming, Z., Hongbin, S. and Ran, A. (2006). A new distributed power flow algorithm between multi-control-centers based on asynchronous iteration, International Conference on Power System Technology .

Iwamoto, S. and Tamura, Y. (1981). A load flow calculation method for ill-conditioned power systems, IEEE Trans. Power App. Syst. (4): 1736-1743.

Kim, B. and Baldick, R. (1997). Coarse-grained distributed optimal power flow, IEEE Trans. Power App. Syst. 12(2): 932-939.

Kim, J., Park, J., Kim, B., Park, J. and Hur, D. (2001). A method of inclusion of security constraints with distributed optimal power flow, Electrical power and energy syst. 23: 189-194.

Kohavi, R. (1995). A study of cross-validation and bootstrap for accuracy estimation and model selection, International Joint Conference on Artificial Intelligence (IJCAI) . http://robotics.stanford.edu/users/ronnyk/.

Nogales, F., Prieto, F. and Conejo, A. (2003). A decomposition methodology applied to the multi-area optimal power flow problem, Annals of operations research (120): 99-116.

Phulpin, Y., Begovic, M., Petit, M., Heyberger, J.-B. and Ernst, D. (2009). Evaluation of network equivalents for voltage optimization in multi-area power systems, IEEE Trans. Power Syst. 24(2): 729-743.

Prechelt, L. P. (1994). A set of neural network benchmark problems and benchmarking rules, Technical Report, Fakultat Fur Informatik pp. 21-94.

Sasson, A. (1969). Nonlinear programming solutions for the load-flow, minimum-loss, and economic dispatching problems, IEEE Trans. Power App. Syst. pp. 399-409.

Sasson, A., Trevino, C. and Aboytes, F. (1971). Improved newton's load flow through a minimization technique, IEEE Trans. Power App. Syst. pp. 1974-1981.

Tomim, M., Martí, J. and Wang, L. (2009). Parallel solution of large power system networks using the multi-area thévenin equivalents (mate) algorithm, Electrical Power and Energy Systems 31: 497-503.

Zhu, J. (2009). Optimization of power system operation, A Jonh Wiley \& Sons, Inc., Hoboken, New Jersey. 